\title{
Spatial Disparity Analysis of Inner Mongolia Based on GIS
}

\author{
Xiaoliang Liu, Zhiyang Jia, Wanli Liu \\ Tourism and Culture College of Yunnan University, \\ Lijiang, China
}

\begin{abstract}
With the application of Geographic Information Systems (GIS) and Exploratory Spatial Data Analysis (ESDA) techniques, twelve criteria indicating the county economic strength of Inner Mongolia are selected to evaluate an analysis and discussed its causes on the spatial disparity. In order to make the factor analysis, principal component analysis (PCA) is performed by the SPSS software. Conclusions concerning global spatial autocorrelation analysis and local spatial autocorrelation analysis are studied. Some significant graphs and charts are demonstrated according to statistics of the results.
\end{abstract}

Keywords-spatial disparity analysis; inner Mongolia; GIS; ESDA; PCA; SPSS

\section{INTRODUCTION}

Regional divergence is a common phenomenon in the regional economic development as well as a core topic of regional economics. The research scale of the regional divergence is relatively distinct of which it is involved with the disparities among the eastern, central and western belts, between the transitional counties and at the county scale. Exploratory Spatial Data Analysis (ESDA) techniques are discussed from the evaluation of single criteria to the evaluation of multi-criteria [1]. Since the county economy plays an important role in government decision-making and macro-control, the research of the disparities at the county scale is significant for the provincial governments and central government to control the regional economic development [2]. Many scholars have applied GIS technology into species distribution, demographic change, botany, regional economy [3], etc.

\section{Methodology}

On the basis of the administrative division of Inner Mongolia in 2010, we chooses 101 counties (banner, city and district) in Inner Mongolia as our study objects, is supported by GIS software platform [4] and takes the SPSS statistical software as the auxiliary tool. With the spatial autocorrelation approach, the frequency map, global and local autocorrelation testing and other tools, the quantitative research is conducted on the spatial disparities of the county economic strength of Inner Mongolia autonomous region.

\section{A. Selection of Evaluation Criteria}

On account of the relatively comprehensive nature of the multi-criteria evaluation, the multiple criteria are applied to evaluate the county economic strength in this paper. The county economic strength is mainly measured by the economic development level at per capita index, reflecting the industrial economic structure and the economic benefit of output-input ratio, etc. The specific indexes are stated as follows: Per Capita Revenue (x1), Average Wages of On-Post Staff (x2), Rural Per Capita Net Income (x3), Per Capita Deposit Balance (x4), Proportion of Output Value of the 2nd Industry (x5), Proportion of Output Value of the 3rd industry (x6), Proportion of Employed Persons of the 2nd Industry (x7), Proportion of Employed Persons of the 3rd industry (x8), Ratio of fiscal Revenue to GDP (x9), Industrial ValueAdded Rate (x10), Per Capita GDP (x11), Total Retail Sales of Social Consumer Goods (x12). Among the twelve criteria, the unit of total retail sales of social consumer goods is one hundred million CNY.

\section{B. Factor Analysis}

The SPSS software is applied to make the factor analysis of which the main approach is Principal Component Analysis (PCA) [5] in making the original multi-criteria $\mathrm{x}$ integrated into one or several indexes that the small amount of indexes will reflect the vast majority of information of multi-criteria.

\section{Spatial Autocorrelation Analysis}

Spatial autocorrelation, as one of the important forms of spatial dependence, refers to the correlation between the research object and the spatial location. Spatial autocorrelation is an important index to verify whether there is a significant association between a particular attribute value and the attribute value of its adjacent element. With the assistance of the Arcview software, it is possible to analyze the spatial autocorrelation.

\section{Data Source}

The selected criteria could be rather complete to reflect the county economic strength. Since the information is acquired from the current official statistics data with the good credibility and integrity. The data originates from the statistical yearbook of Inner Mongolia. The spatial analysis scale is 101 counties (banner, city and district) in the Inner Mongolia autonomous region, and the administrative boundary data is taken from the administrative division map of Inner Mongolia at the proportion of 1: 10000000 based on vector quantization of scanning the tracks. 


\section{COUNTY ECONOMIC STRENGTH EVALUATION}

The principal component analysis (PCA) approach is carried out by SPSS. We can find that $\mathrm{KMO}=0.7$ among the 12 criteria, which indicating the correlation among the variables and the rightness of making the factor analysis. According to the election principle that the principal divisor of the eigenvalues is greater than 1 , the selected eigenvalues were respectively 4.307, 2.383 and 1.130. At the same time, the cumulative variance contribution is $65.169 \%$, which the majority of information is included (Table I). Based on the clearer component matrix (Table II) obtained from the orthogonal rotation of the varimax method, the first principal component F1 is called after economic development factor, the second principal component F2 is called after economic structure factor and the third principal component F3 is called after economic benefit factor.

TABLE I. FACTOR EIGENVALUES AND CONTRIBUTION RATE

No. Eigenvalue Contribution rate (\%) Accumulative contribution rate (\%)

\begin{tabular}{|c|c|c|c|}
\hline 1 & 4.307 & 35.888 & 35.888 \\
\hline 2 & 2.383 & 19.860 & 55.748 \\
\hline 3 & 1.130 & 9.421 & 65.169 \\
\hline 4 & 0.898 & 7.486 & 72.655 \\
\hline 5 & 0.827 & 6.894 & 79.549 \\
\hline 6 & 0.677 & 5.638 & 85.187 \\
\hline 7 & 0.529 & 4.405 & 89.591 \\
\hline 8 & 0.418 & 3.486 & 93.077 \\
\hline 9 & 0.364 & 3.035 & 96.112 \\
\hline 10 & 0.248 & 2.068 & 98.180 \\
\hline 11 & 0.169 & 1.410 & 99.590 \\
\hline 12 & 0.049 & 0.410 & 100.00 \\
\hline
\end{tabular}

By using the regression method [6], we can calculate each factor score and economic strength score F, which the respective contribution rates of the first principal component F1, the second principal components F2 and the third principal component F3 are their corresponding weights of the three principal components. The contribution rate of the first principal component F1 is 0.35888 with the contribution rate of the second principal component F2 at 0.19860 and the contribution rate of the third principal component F3 at 0.09421. In this way, the man-made factor on the scoring preferences of the experts is restricted. Just given the pure number, the scoring model of the county economic strength shall be $F=0.35888^{*} F 1+0.19860 * F 2+0.09421 * F 3$. We can calculate the economic strength score for the counties of Inner Mongolia autonomous region. With the higher scores, the county has the higher level of economic strength, otherwise vice versa. The fact that the score is greater than 0 indicates that the level of economic strength is higher than the average level of the whole autonomous region, and the score is less than 0 indicates that the level of economic strength is lower than the average level. 
TABLE II. MATRIX OF PRINCIPAL COMPONENT ANALYSIS

\begin{tabular}{lccc}
\hline \multicolumn{1}{c}{ Variables } & 1st & 2nd & 3rd \\
\hline Per Capita Revenue (X1) & .90 & -.01 & .02 \\
Average Wages of On-Post Staff (X2) & .81 & .20 & -.02 \\
Rural Per Capita Net Income (X3) & .51 & .31 & .51 \\
Per Capita Deposit Balance (X4) & .39 & .07 & .10 \\
Proportion of Output Value of the 2nd Industry (X5) & .59 & -.58 & .19 \\
Proportion of Output Value of the 3rd industry (X6) & -.01 & .89 & -.18 \\
Proportion of Employed Persons of the 2nd Industry (X7) & .67 & .25 & -.11 \\
Proportion of Employed Persons of the 3rd industry (X8) & .37 & .72 & .06 \\
Ratio Of Fiscal Revenue To GDP (X9) & .75 & -.08 & -.15 \\
Industrial Value-Added Rate (X10) & -.09 & -.11 & .86 \\
Per Capita GDP (X11) & .86 & .051 & .24 \\
Total Retail Sales Of Social Consumer Goods (X12) & .11 & .81 & .15 \\
\hline
\end{tabular}

TABLE III. FREQUENCY DISTRIBUTION CHARACTERISTICS OF THE ECONOMIC STRENGTH SCORES

\begin{tabular}{cc}
\hline Eigenvalues & Value \\
\hline Median & -0.063800 \\
Mode & -0.3922 \\
Std. Deviation & 0.4208488 \\
Skewness & 0.651 \\
Kurtosis & -0.308 \\
Range & 1.7314 \\
Minimum & -0.6144 \\
Maximum & 1.1170 \\
\hline
\end{tabular}

The frequency distribution characteristics based on the scores of the county economic strength represent the economic strength for the 101 counties in Inner Mongolia. From Figure 1 and Table III, we can find that the frequency distribution based on the county economic strength score of Inner Mongolia has a positive skewness with the larger value of skewness at 0.651 of which the data is deviated from the central line with the obviously right skewed distribution. The result shows that the counties with the lower scores are overwhelming. Table 3 illustrates the kurtosis coefficient is -0.308 , which it shows that the gradient of the frequency distribution is lower and evener than that of Gaussian distribution. The low-lying distribution also suggests that the counties with the higher scores occupy a larger proportion. Table 3 illustrates the median is -0.0638 which is slightly less than the mean value 0 . The range is greater at 1.7314 . The value of mode is -0.3922 with the relatively greater standard deviation at 0.4208488 .

\section{Frequency Distribution}

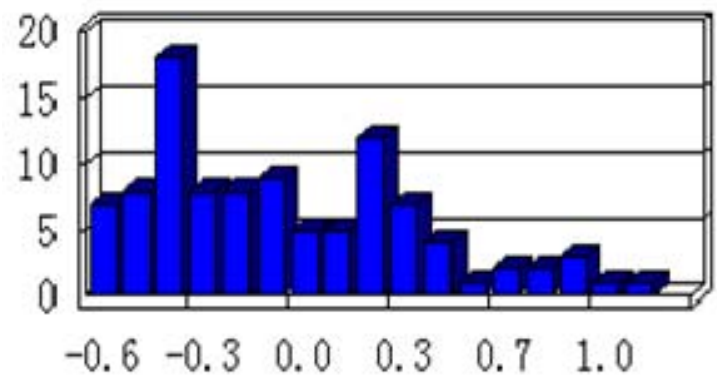

Figure 1. Frequency distribution of the county economic strength scores

The comprehensive scores of each county economic strength and GIS graphic data are linked together. Through the GIS-based statistical analysis, the county economic development levels from 101 counties in Inner Mongolia are classified into four different types (Figure 2): developed areas (The economic strength score is more than 0.7 ), the secondarily developed areas (The economic strength score is greater than 0.5 and less than 0.7 ), the less developed regions (The economic strength score is greater than 0.2 and less than 0.5 ) and the undeveloped area (The economic strength score is below 0.2 ). 


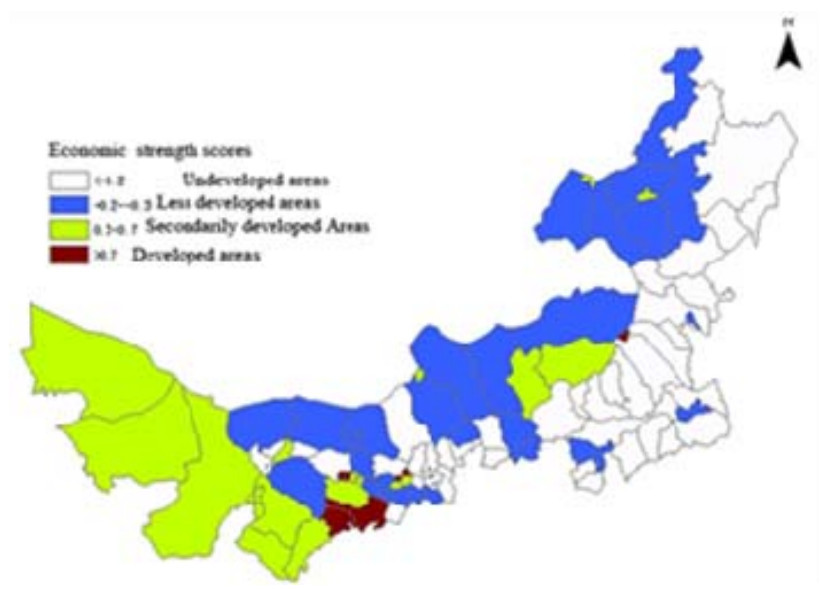

Figure 2. Spatial distribution maps of the county economic strength scores

\section{SPATIAL AUTOCORRELATION ANALYSIS}

\section{A. Spatial Weight Matrix}

During the process of conducting the spatial autocorrelation analysis [7], above of all, it needs to define the mutual adjacency relation of the spatial object and determine the weight of each space unit so as to understand the spatial linkage of the related data in the GIS database. The definition of the spatial weight matrix depends on the adjacency rule and the distance rule. In this paper, the adjacency rule is adopted. Following the adjacency rule, a binary symmetric spatial weight matrix $W$ is used to express the spatial adjacency relationship in the $\mathrm{n}$ position. When $i$ and $j$ is adjacent, the equation of the element in the spatial weight matrix is $W=1$, otherwise $W=0$.

\section{B. Global Spatial Autocorrelation Analysis}

The purpose of global spatial autocorrelation analysis is to explore the spatial distribution characteristic of the attribute data values in the overall area. Through the global spatial autocorrelation statistics of the value estimate of Global Moran's I [8], it is available to analyze the spatial correlation and spatial disparity degree in the overall area.

$$
I(\mathrm{~d})=\frac{\sum_{i}^{n} \sum_{j \neq i}^{n} W_{i j}\left(x_{i}-\bar{x}\right)}{S^{2} \sum_{i}^{n} \sum_{j \neq i}^{n} W_{i j}}
$$

Where $s^{2}=\frac{1}{n} \sum_{i}^{n}\left(x_{i}=\bar{x}\right)^{2}, x^{i}$ denotes the attribute value at $I$, and $\bar{x}$ is the $x$ arithmetic mean value, $W_{i j}$ is spatial weight value. The value range of Moran's $I$ statistics is generally from -1 to 1 . If $I(d)<0$, then it means that the space has a negative correlation, indicating the significant spatial disparity of the economic strength between the region and its surrounding areas. As the value is approaching to -1 , the overall spatial disparity is greater. If $I(d)>0$, then the space has a positive correlation, indicating that the accumulative degree is significant for the areas with the higher (lower) economic strength. As the value is approaching to 1 , the overall spatial disparity is smaller. If $I(d)=0$, then it means the space is not associated. If $C>1$ (Geary coefficient), then it has a negative correlation. If $C=1$, then it means the there is no association. If $C<1$, then it means the positive correlation. By (1), the value of Moran's I of the global autocorrelation coefficient is 0.423631 in terms of the county economic strength in the Inner Mongolia autonomous region, which the value is greater than 0 while the Geary coefficient is less than 1 at 0.50837 . The result shows that the county economic strength of Inner Mongolia has a positive correlation.

For the Moran index [9], a standardized statistics $Z$ can be applied to test the spatial autocorrelation in the 101 counties (cities, districts) of Inner Mongolia. If the correlation of $Z$ is positively significant, it suggests there is positively spatial autocorrelation. As well, the observed value (high value or low value) tends to be spatial agglomeration. When the correlation of $Z$ is negatively significant, it suggests there is negatively spatial autocorrelation. The distribution of the similar observations tends to be scattered, such as the agglomeration of the high and low observation value. If $Z$ equals 0 , then the observed value is independently and randomly distributed. By our calculation, $Z=6.16204$ is obtained. The result (as shown in Table IV) indicates that there is positively spatial autocorrelation for the county economic strength in Inner Mongolia, namely the area with the high economic strength or the area with the low economic strength tends to be the spatial agglomeration.

TABLE IV. GLOBAL StATISTICS

\begin{tabular}{lccc}
\hline & Statistics & Expectation & Z-value \\
\hline Moran's I & 0.423631 & -0.01 & 6.16204 \\
Geary's $C$ & 0.50837 & 1 & -6.56744 \\
G-statistics & $9.85053 \mathrm{e}-005$ & 0.00019802 & -0.490725 \\
\hline
\end{tabular}




\section{Local Spatial Autocorrelation Analysis}

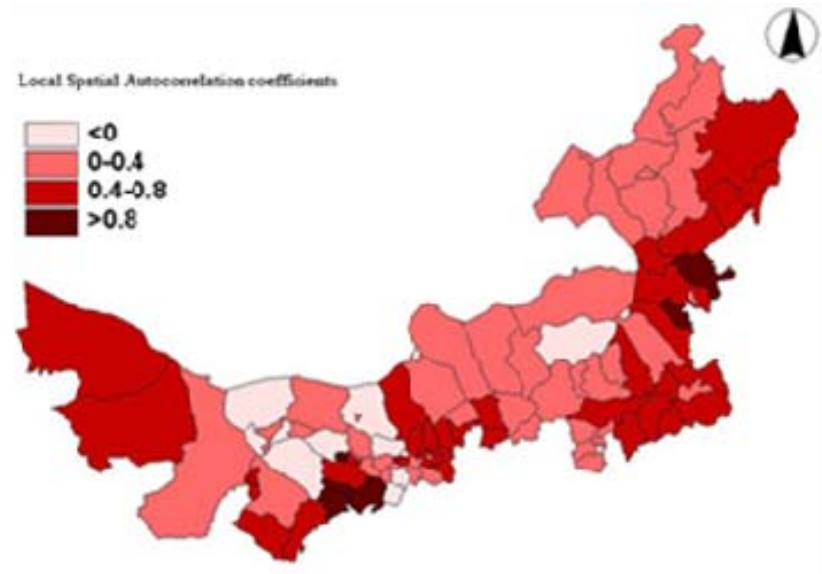

Figure 3. Distribution of local spatial autocorrelation coefficients

The local spatial autocorrelation analysis majorly focuses on the distribution pattern of the attribute value of each unit in the heterogeneous space so as to measure the local spatial correlation degree between each region and its surrounding areas. The commonly used statistics is the Local Moran's $I_{i}$, measured by

$I_{i}=Z_{j} \sum_{j \neq i}^{n} W_{i j Z_{j}}$

Where $Z_{i}$ and $Z_{j}$ are the standardized forms of the element attribute values, and $W_{i j}$ is spatial weight matrix. If $I_{i}$ is significantly greater than 0 , it means that the space is slightly different between the area $i$ and the surrounding areas. If $I_{i}$ is significantly less than 0 , it means that the space is significantly different between the area $i$ and the surrounding areas. (2) is used to calculate Local Moran's $I_{i}$ of the local spatial autocorrelation coefficient for each county unit of Inner Mongolia. Afterwards, the calculated results are linked with the corresponding graphical data to draw the LSIA map and generate the distribution diagram of the local spatial autocorrelation coefficient (As shown in Fig. 3) based on the GIS thematic mapping function.

\section{REFERENCES}

[1] Guillain R, Le Gallo J. Agglomeration and dispersion of economic activities in and around Paris: an exploratory spatial data analysis. Environment and Planning B: Planning and Design, 37(6) 961-981, 2009.

[2] Hualin Xie, Chih-Chun Kung, Yuluan Zhao. Spatial disparities of regional forest land change based on ESDA and GIS at the county level in Beijing-Tianjin-Hebei area. Frontiers of Earth Science, 6(4), pp 445-452, 2012.

[3] David M. Theobald,Don L. Stevens Jr.,Denis White,et al. Using GIS to Generate Spatially Balanced Random Survey Designs for Natural Resource Applications. Environmental Management, 40(1), pp 134-146, 2007.

[4] Jin Luo, Dian-Hong Wang, et al. Popularized Spatial Information Service Platform Based on Multi-Major GIS, Second International Workshop on Knowledge Discovery and Data Mining, pp 890-894, 2009.

[5] Lihua Zhao, M. Uncles, G. Gregory, null Hongwei Lu, A. Mann. GIS and spatial information in business decision support: the case of automobile service network planning, International Conference on Services Systems and Services Management, pp.1157-1161 Vol. 2, 2005.

[6] Guangqing Chi,Jun Zhu. Spatial Regression Models for Demographic Analysis.Population Research and Policy Review, 27(1), pp 17-42, 2008.

[7] Chen Fei, Du Daosheng. Application of integration of spatial statistical analysis with GIS to regional economic analysis. Geospatial Information Science, 7(4), pp 262-267, 2004.

[8] L Anselin. The Moran scatterplot as an ESDA tool to assess local instability in spatial association, Spatial Analytical Perspectives on GIS, Taylor \& Francis Inc, 1996.

[9] Yang Yang, Kevin K. F. Wong. Spatial Distribution of Tourist Flows to China's Cities. Tourism Geographies: An International Journal of Tourism Space, Place and Environment, 15(2), pp 338-363, 2013. 